\title{
Determination of Angiopoietin Like Protein-4 Levels and Some Parameters in Iraqi Patients with Type 2 Diabetes Mellitus
}

\author{
Shatha Abdul Wadood*, Yahya D. Saihood ${ }^{* *}$ and Zahraa J. Mohammed** \\ *Department of Chemistry, College of Science, University of Baghdad, Baghdad-Iraq. \\ ${ }^{* *}$ National Diabetes Center, Al-Mustansiyria University, Baghdad-Iraq.
}

\begin{abstract}
A. Background: Diabetes initiates the thrombotic complications of atherosclerosis through changing the function of endothelial cells, smooth muscle cells and platelets. Also insulin resistance, hyperglycemia and dyslipidemia can lead to arterial atherosclerosis in diabetic patients. Angiopoietin like protein- 4 (ANGPTL4) is a multifunctional protein involved in lipid regulation, energy metabolism, angiogenesis, and inflammation. Its expression is stimulated in liver, heart, muscle and adipose tissue, during the acute phase response. C-reactive protein directly induces the expression of adhesion molecules by endothelial cells. Also recruiting monocytes into the vessel wall are triggered by the expression of specific chemokines particularly monocyte chemoattractant protein-1 (MCP-1), all these processes induce the risk of atherosclerotic complications with type 2 diabetes mellitus (T2DM).

B. Aim of this study: To study the role of ANGPTL4 in T2DM patients with and without atherosclerosis risk factor (dyslipidemia), and to demonstrate the relationship of ANGPTL4 with inflammatory process.
\end{abstract}

Keywords: Angiopoietin like protein- 4; Dyslipidemia; Type 2 diabetes mellitus; Inflammation.

\section{Introduction}

Type 2 Diabetes Mellitus is a chronic condition which is considered as a major cause of mortality and morbidity for its microvascular (retinopathy, nephropathy and neuropathy) and macro-vascular (coronary heart disease, peripheral vascular disease and stroke) complications. It is a multi-factorial disease that in its development, in addition to genetic predisposition, a number of environmental factors such as poor nutrition, lack of physical activity and obesity play major roles. Among these factors, obesity, dyslipidemia and hypertension have more potent relationship with T2DM which may link the ANGPTL4 and in flammatory marker to diabetic complications [1]. Cardiovascular disease CVD (broadly defined as stroke, coronary artery disease, and peripheral vascular disease) is the major cause of morbidity and mortality in patients with T2DM. CVD leads to $65 \%$ of deaths in diabetic patients. According to the updated US National Cholesterol Education Program (NCEP) Adult Treatment Panel (ATP) III guidelines, T2DM patients are categorized as having the highest level of risk for recurrent CVD events and they are in the same risk category as patients with acute coronary syndrome (ACS) [2].

Inflammation plays a significant role in both CVD and T2DM. Inflammation can lead to atherogenesis, atheromatous plaque rupture, and thrombus formation that cause ACS [3].

ANGPTL-4 is a multifunctional protein involved in lipid regulation, energy metabolism, angiogenesis, and inflammation. Its expression is stimulated in liver, heart, muscle and adipose tissue, during the acute phase response; it is an endogenous inhibitor of the triglyceride-hydrolyzing enzyme lipoprotein lipase (LPL) that catalyzes uptake of circulating lipids into tissues [4]. Irreversible inhibition of LPL activity by ANGPTL4 through converting active LPL dimer into inactive monomers consequently, overexpression of ANGPTL4 leads to hypertriglyceridemia and reduced fatty acid uptake in tissues, whereas ANGPTL4 deletion causes lowering of circulating triglyceride levels [5]. By inhibiting LPL activity, ANGPTL4 was previously shown to reduce macrophage uptake of triglycerides-derived fatty acids and impair macrophage activation [6], which may indirectly lead to decreased uptake of oxidized LDL [7]. C-Reactive Protein can induce the expression of tissue 
factor by monocytes, and it is present in atherosclerotic plaques but not in the normal vessel wall $[7,8]$. CRP has several direct effects that may affect vascular disease progression. These reported functions include an ability to bind and activate complement, induce expression of several cell adhesion molecules as well as tissue factor, mediate LDL uptake by endothelial macrophages, induce monocyte recruitment into the arterial wall, and enhance production ofmonocyte chemoattractant protein-1 MCP-1 [9].

\section{Subjects and Methods}

\section{A. Subjects:}

This study was conducted on Iraqi patients, 24 T2DM patients without atherosclerosis risk factor (dyslipidemia) group $\mathrm{P} 1$; duration of diabetes was less than 5 years) and 30 T2DM patients with atherosclerosis risk factor (dyslipidemia), (group P2; duration of diabetes was more than 10 years) who attended to the National Diabetes Center of Al- Mustansiyria University, as well as 26 non diabetic healthy persons to serve as control (Group C). All subjects were informed of the purpose of the study and their consent was obtained. T2DM is diagnosed and classified according to the Expert Committee on the Diagnosis and Classification of Diabetes Mellitus [10]. A detailed history and complete clinical examination were done. All demographic and clinical data of subjects were collected in the form of age and gender. Their weight, height, waist to hip ratio (WHR), waist to height ratio (WHtR), and body mass index (BMI) were calculated.

Dyslipidemia is change in plasma level of lipid elevated in $\mathrm{TG}(\mathrm{TG} \geq 150 \mathrm{mg} / \mathrm{dl})$ and decreased in level of HDL-C (HDL-C $\leq 40 \mathrm{mg} / \mathrm{dl}$ ).

Systolic and diastolic blood pressures were recorded as a mean of three successive measurements. Group $\mathrm{C}$ was judged to be in good health according to their medical history and physical examination as well as their fasting and postprandial blood glucose. All T2DM patients were under treatment with Metformin (Glucophage) or a combination of Glibenclamideknown as sulfonylureas (Daonil) and Metformin as glucose lowering drugs.
B. Exclusion criteria were: Cushing's disease, acromegaly, chronic pancreatitis, pancreatactomy, pregnancy, history or even manifestation ofnephropathy, chronic renal failure, malignancies and chronic or acute inflammatory disease, patients who were taking aspirin, lipid lowering therapy and insulin therapy, history of smoking or alcohol drinking were excluded.

C. Sampling: Blood samples were drawn from subjects on overnight fasting (12hours). All blood samples were divided into 2 tubes: and divided in to 2 and $8 \mathrm{ml}$; one with anticoagulant to aspirated plasma and the second without any anticoagulant (tubes gel) were put in centrifuge for 10 minutes after clotting; then serum was aspirated and divided into aliquots $(250 \mu 1)$ in Eppendorff tubes, and stored in deep freeze $\left(-20^{\circ} \mathrm{c}\right)$ till used.

D. Laboratory investigations: Serum total cholesterol (TC) and triglyceride (TG) were analyzed using fully enzymatic methods with commercially available kits (SPINREACT, S.A/S.A.U, Spain). Serum HDL -cholesterol (HDL-C) was measured using direct enzymatic methods (RANDOX, United Kingdom) and serum LDL-cholesterol (LDLC) and (VLDL) were calculated using the Friedewald formula (11). [LDL-C $(\mathrm{mg} / \mathrm{l})=\mathrm{TC}$ $-(\mathrm{TG} / 5+\mathrm{HDL})], \mathrm{VLDL}(\mathrm{mg} / \mathrm{l})=\mathrm{TG} / 5$

Atherogenic index of plasma (AIP) was calculated by the equation:

$\mathrm{AIP}=\log (\mathrm{TG} / \mathrm{HDL}-\mathrm{C})[12]$.

Risk Ratio1 (RR1) was obtained by dividing total cholesterol by HDL-cholesterol (TC/ HDL-C) and risk Ratio2 (RR2) was obtained by dividing LDL-cholesterol by HDL-cholesterol (LDL-C/ HDL-C), and these ratios are considered the greatest valuable for evaluation of CVD risk (National Cholesterol Education Program, 1994 and Grundy, 1989). Fasting Plasma glucose (FPG) concentration was analyzed by a hexokinase method (Spinreact, S.A.U, Spain), and insulin was analyzed by ELISA (DRG instruments $\mathrm{GmbH}$, Germany). Concentration of ANGPTL4, MCP-1 and C-reactive protein (CRP) were determined by ELISA method (Ray Biotech, Inc. USA).The intra- and inter-assay coefficients of variation for the assays were 
less than $10 \%$ and $12 \%$ respectively. Computer-based Homeostasis Model Assessment (HOMA) of insulin resistance (HOMA IR) and insulin sensitivity was previously detailed (http://www.dtu.ox.ac.uk).

E. Statistical analyses: A one-way analysis of variance (ANOVA) and multiple comparison were used to determine the difference between various groups .The results are expressed as Mean \pm Standard deviation (SD.). Bivariate Pearson's product correlation coefficient(r) was used to test the strength of association between serum (ANGPTL4, MCP-1and hsCRP) levels and other variables in the three groups (P1 and P2). Simple scatter plot was used to illustrate the significant correlation, while simple bar chart was used to show the significant differences of ANGPTL4, MCPland hs-CRP between groups. All statistical analyses were performed by using SPSS 17.0 (SPSSInc., Chicago, IL, USA). Differences were considered statistically significant at $\mathrm{p}<0.05$ and highly significant at $\mathrm{p}<0.01$.

\section{Results}

The results showed that significant increase in waist, WHR, WHtR, FPG, Insulin, HOMA2 parameters (IR, S\%, and $\beta \%$ ), VLDL, AIP, MCP-1 and hs-CRP was found in group (P1) in comparison with group (C), while ANGPTL-4 showed significant decrease in group (P1) in comparison with group (C) (Table (1)) (Fig.(1)).

Table (1) shows significant increase in duration of diabetes, diastolic B P, FPG, TC, TG, LDL, VLDL, AIP, RR1and RR2 of diabetes patients group (P2) in compression with group $(\mathrm{P} 1)(\mathrm{P}<0.001)$, while HDL shows significant decrease. Also ANGPTL-4, MCP1, hs-CRP (Fig.(1)), BMI, smoking, SBP and HOMA2 $-\beta \%$ was significantly increased in group (P2) in comparison to group (P1) $(\mathrm{P}<0.05)$.However, age, weight, height, waist, hip, WHR, WHtR, F.P.G, Insulin, HOMA2-

IR and HOMA2 S\% showed no significant differences between the two groups (P1 and P2) (P> 0.05) Table (1).

Also, the results showed positive correlation between ANGPTL-4 and hs-CRP in diabetes patients group (P1) Table (2), Fig.(2). ANGPTL-4 of group (P2) showed positive correlations with WHR, TC, TG, VLDL, AIP, RR1and RR2, and negatively correlated with hs-CRP Table (3), Fig.(3).

MCP-1 shows positive correlation with TG and VLDL of diabetes patients group (P1) (Table (2)), and a positive correlation with TC, LDL and RR2 of diabetes patients group (P2) Table (3).

hs-CRP shows positive correlation with Insulin and HOMA2 I.R in both patients groups (Table2). Also hs-CRP in group (P2) shows positive correlation with insulin, age, waist, hip and WHtR Table (3). 
Table (1)

Mean and STD of Various parameters for studied groups.

\begin{tabular}{|c|c|c|c|c|}
\hline Parameter & $\begin{array}{c}\text { Group }(C) \\
n=26 \\
\text { (mean } \pm S T D)\end{array}$ & $\begin{array}{c}\text { Group (P1) } \\
n=24 \\
\text { (mean } \pm \text { STD) }\end{array}$ & $\begin{array}{c}\text { Group (P2) } \\
n=30 \\
\text { (mean } \pm \text { STD) }\end{array}$ & $\begin{array}{c}\text { Pvalue } \\
\text { using } \\
\text { ANOVA }\end{array}$ \\
\hline Number (male/female) & $(14 / 12)$ & $(19 / 4)$ & $(17 / 13)$ & - \\
\hline Age (year) & $36-63$ & $36-65$ & $42-64 * b$ & 0.102 \\
\hline $\begin{array}{l}\text { Duration of diabetes } \\
\text { (year) }\end{array}$ & - & $2.58 \pm 1.71$ & $11.46 \pm 1.79 * * \mathrm{c}$ & - \\
\hline Weight(Kg) & $75.27 \pm 8.34$ & $79.17 \pm 7.48$ & $78.77 \pm 9.04$ & 0.192 \\
\hline Height ( Cm) & $169.15 \pm 7.97$ & $168.88 \pm 5.77$ & $168.3 \pm 7.29$ & 0.900 \\
\hline $\mathrm{BMI}\left(\mathrm{kg} / \mathrm{m}^{2}\right)$ & $26.3 \pm 1.67$ & $27.22 \pm 1.99$ & $28.42 \pm 1.92 * \mathrm{~b} \mathrm{c}$ & $<<0.01$ \\
\hline Waist $(\mathrm{Cm})$ & $95.73 \pm 6.40$ & $100.33 \pm 6.07 * a$ & $101.43 \pm 8.77 * \mathrm{~b}$ & 0.130 \\
\hline $\operatorname{Hip}(\mathrm{Cm})$ & $102.85 \pm 4.91$ & $101.92 \pm 5.74$ & $102.77 \pm 8.87$ & 0.869 \\
\hline WHR & $0.926 \pm 0.04$ & $0.98 \pm 0.04^{* a}$ & $0.98 \pm 0.02 * \mathrm{~b}$ & $<0.01$ \\
\hline WHtR & $0.561 \pm 0.04$ & $0.59 \pm 0.04^{* a}$ & $0.602 \pm 0.05 * \mathrm{~b}$ & <<0.01 \\
\hline Smoking & 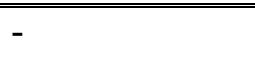 & $1.13 \pm 0.33$ & $1.4 \pm 0.49 * \mathrm{~b}_{\mathrm{c}}$ & 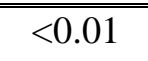 \\
\hline Systolic B.P.(mm Hg) & $124.23 \pm 5.03$ & $124.58 \pm 6.50$ & $138 \pm 17.69 * \mathrm{~b}_{\mathrm{c}}$ & 0.311 \\
\hline Diastolic B.P. (mmHg) & $76.92 \pm 4.70$ & $78.33 \pm 3.80$ & $84.67 \pm 5.71 * \mathrm{~b} * * \mathrm{c}$ & 0.136 \\
\hline FPG (mg/dl) & $87.46 \pm 7.96$ & $158.708 \pm 47.9 * a$ & $224.26 \pm 72.82 * \mathrm{~b} * *$ & $<0.01$ \\
\hline Insulin $(\mu \mathrm{IU} / \mathrm{ml})$ & $6.37 \pm 4.85$ & $15.94 \pm 10.64 * a$ & $10.75 \pm 11.94 * b$ & $<0.01$ \\
\hline HOMA2-IR & $0.93 \pm 0.73$ & $2.26 \pm 1.40 * \mathrm{a}$ & $1.99 \pm 2.26 * \mathrm{~b}$ & 0.012 \\
\hline HOMA2 (S\%) & $147.13 \pm 68.25$ & $63.79 \pm 50.76 * a$ & $82.23 \pm 36.59 * \mathrm{~b}$ & $<<0.01$ \\
\hline HOMA2 (B\%) & $95.08 \pm 51.10$ & $64.65 \pm 44.85 * a$ & $26.62 \pm 26.52 * b_{c}$ & $2<0.01$ \\
\hline TC (mg/dl) & $148.84 \pm 18.85$ & $157.25 \pm 22.94$ & $225.8 \pm 33.28 * \mathrm{~b} * * \mathrm{c}$ & $<<0.01$ \\
\hline TG(mg/dl) & $92.5 \pm 17.23$ & $118.79 \pm 26.29 * a$ & $218.6 \pm 134.09 * \mathrm{~b} * *$ & $<<0.01$ \\
\hline HDL (mg/dl) & $54.03 \pm 8.43$ & $51.75 \pm 7.09$ & $39.16 \pm 5.33 * * \mathrm{~b} * * \mathrm{c}$ & $<0.01$ \\
\hline LDL (mg/dl) & $77.3 \pm 18.01$ & $82.37 \pm 20.91$ & $139.56 \pm 22.48 * \mathrm{~b} * *$ & $<0.01$ \\
\hline VLDL (mg/dl) & $18.96 \pm 4.47$ & $23.79 \pm 5.18 * a$ & $43.73 \pm 26.84 * \mathrm{~b} * * \mathrm{c}$ & $<0.01$ \\
\hline AIP & $0.236 \pm 0.09$ & $0.35 \pm 0.13 * a$ & $0.65 \pm 0.3 * \mathrm{~b} * * \mathrm{c}$ & $<0.01$ \\
\hline RR1 & $2.82 \pm 0.48$ & $3.09 \pm 0.57$ & $5.47 \pm 1.34 * \mathrm{~b} * * \mathrm{c}$ & $<0.01$ \\
\hline RR2 & $1.457 \pm 0.41$ & $1.6 \pm 0.48$ & $3.337 \pm 0.67 * \mathrm{~b} * * \mathrm{c}$ & $<<0.01$ \\
\hline ANGPTL-4(ng/ml) & $3.08 \pm 0.93$ & $2.60 \pm 0.72 * a$ & $5.41 \pm 5.11 * \mathrm{~b} * * \mathrm{c}$ & $<0.01$ \\
\hline MCP-1(pg/ml) & $121.34 \pm 54.50$ & $219.1 \pm 47.98 * a$ & $254.7 \pm 63.12 * \mathrm{~b} * * \mathrm{c}$ & $<<0.01$ \\
\hline hs-CRP(mg/l) & $1.443 \pm 0.72$ & $2.142 \pm 1.53 * a$ & $2.935 \pm 1.14 * \mathrm{~b} * * \mathrm{c}$ & $<0.01$ \\
\hline
\end{tabular}

**: $P<0.01, *: P<0.05$. a: significant between $P 1 \& C, b$ : significant between $P 2 \& C$, $c:$ significant between P1 \& P2, using multiple comparison. 
Table (2)

Pearson's correlation between fasting ANGPTL4, MCP-1 and hs-CRP with all parameters in diabetic patients (group P1).

\begin{tabular}{|c|c|c|c|c|c|c|c|c|c|}
\hline \multirow{2}{*}{ Parameter } & \multicolumn{3}{|c|}{ ANGPTL-4(ng/ml) } & \multicolumn{3}{|c|}{$M C P-1(p g / m l)$} & \multicolumn{3}{|c|}{$\overline{C R P(m g / l)}$} \\
\hline & $R$ & P value & Sig. & $R$ & Pvalue & Sig. & $R$ & P value & Sig. \\
\hline Age(year) & -0.273 & 0.265 & NS & -0.222 & 0.239 & NS & -0.163 & 0.445 & $\mathrm{NS}$ \\
\hline $\begin{array}{l}\text { Duration of disease } \\
\text { (year) }\end{array}$ & 0.147 & 0.493 & NS & 0.258 & 0.224 & NS & -0.317 & 0.131 & NS \\
\hline Weight $(\mathrm{Kg})$ & 0.240 & 0.258 & NS & -0.037 & 0.868 & NS & 0.059 & 0.786 & NS \\
\hline Height $(\mathrm{cm})$ & 0.167 & 0.435 & NS & 0.003 & 0.988 & NS & $0.113-$ & 0.601 & NS \\
\hline $\mathrm{BMI}\left(\mathrm{Kg} / \mathrm{m}^{2}\right)$ & 0.207 & 0.331 & NS & -0.213 & 0.317 & NS & 0.129 & 0.547 & $\mathrm{NS}$ \\
\hline Waist(cm) & 0.163 & 0.446 & NS & 0.212 & 0.319 & NS & 0.074 & 0.730 & NS \\
\hline $\operatorname{Hip}(\mathrm{cm})$ & 0.078 & 0.719 & NS & -0.114 & 0.596 & NS & 0.129 & 0.546 & $\mathrm{NS}$ \\
\hline "WHR & 0.176 & $\overline{0.412}$ & NS & 0.254 & 0.230 & NS & $\begin{array}{c}-0.064 \\
\end{array}$ & 0.766 & NS \\
\hline$\overline{\overline{\text { WHtR }}}$ & 0.014 & 0.948 & NSS & 0.039 & 0.856 & NS & 0.105 & 0.626 & $\overline{\mathrm{NS}}$ \\
\hline Smoking & 0.209 & 0.326 & NS & -0.137 & 0.525 & NS & 0.171 & 0.425 & NS \\
\hline $\begin{array}{l}\text { Systolic B.P. } \\
(\mathrm{mm} \mathrm{Hg})\end{array}$ & -0.398 & 0.115 & NS & -0.115 & 0.593 & NS & 0.222 & 0.298 & NS \\
\hline $\begin{array}{l}\text { Diastolic B.P. } \\
(\mathrm{mmHg})\end{array}$ & -0.04 & 0.851 & NS & -0.210 & 0.325 & NS & 0.226 & 0.287 & NS \\
\hline FPG (mg/dl) & -0.084 & 0.696 & NS & -0.391 & 0.059 & NS & 0.081 & 0.707 & NS \\
\hline F.P. Insulin $(\mu \mathrm{IU} / \mathrm{ml}$ & 0.261 & 0.218 & NS & -0.230 & 0.279 & NS & 0.538 & 0.007 & $\bar{S}$ \\
\hline HOMA2 (I.R) & 0.289 & 0.171 & NS & -0.263 & 0.214 & NS & 0.568 & 0.004 & $S$ \\
\hline HOMA2 (S\%) & -0.259 & 0.221 & NS & 0.215 & 0.313 & NS & -0.314 & 0.135 & $\mathrm{NS}$ \\
\hline HOMA2 (B\%) & 0.204 & 0.34 & NS & $\begin{array}{c}0.118 \\
\end{array}$ & 0.583 & NS & 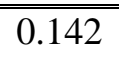 & 0.509 & $\overline{\mathrm{NS}}$ \\
\hline TC (mg/dl) & "-0.151 & 0.48 & NS & 0.193 & 0.367 & $\overline{\mathrm{NS}}$ & -0.323 & 0.09 & $\overline{\mathrm{NS}}$ \\
\hline TG(mg/dl) & $\begin{array}{c}-0.043 \\
\end{array}$ & $\overline{0.842}$ & $\overline{\mathrm{NSS}}$ & 0.426 & 0.038 & $\overline{S S}$ & $2-0.275$ & 0.193 & $\overline{\mathrm{NS}}$ \\
\hline "HDL (mg/dl) & $\begin{array}{l}-0.09 \\
\end{array}$ & 0.677 & NS & 0.153 & 0.475 & NS & 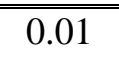 & 0.962 & $\overline{\mathrm{NS}}$ \\
\hline$\overline{\mathrm{LDL}}(\mathrm{mg} / \mathrm{dl})$ & -0.128 & 0.55 & $\overline{\mathrm{NSS}}$ & 0.129 & 0.549 & $\overline{\mathrm{NS}}$ & -0.371 & 0.074 & NS \\
\hline VLDL (mg/dl) & -0.027 & 0.899 & NS & 0.432 & 0.035 & $\bar{S}$ & -0.264 & 0.212 & $\overline{\mathrm{NS}}$ \\
\hline AIP & 0.001 & 0.995 & $\overline{\mathrm{NS}}$ & 0.32 & 0.127 & $\overline{\mathrm{NS}}$ & $2-0.232$ & 0.276 & NS \\
\hline RR1 & -0.056 & 0.796 & $\overline{\mathrm{NSS}}$ & 0.131 & 0.543 & $\overline{\mathrm{NS}}$ & $2-0.189$ & 0.377 & NS \\
\hline RR2 & $\begin{array}{l}-0.092 \\
\end{array}$ & 0.669 & NS & 0.16 & 0.454 & NS & -0.182 & 0.394 & NS \\
\hline ANGPTL- 4(ng/ml) & 1.00 & - & 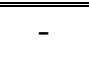 & 0.055 & 0.799 & NS & 0.417 & 0.04 & $\overline{\mathrm{S}}$ \\
\hline MCP-1(pg/ml) & 0.055 & 0.799 & NS & $\bar{~} 1$ & - & - & $\begin{array}{c}-0.311 \\
\end{array}$ & $\begin{array}{l}0.139 \\
\end{array}$ & $\overline{\mathrm{NS}}$ \\
\hline$\overline{\mathrm{hs}-\mathrm{CRP}(\mathrm{mg} / \mathrm{l})}$ & 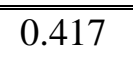 & 0.04 & $\bar{S}$ & 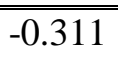 & 0.139 & $\overline{\mathrm{NSS}}$ & 1 & - & - \\
\hline
\end{tabular}

H.S.: $P<0.001$, S. :P<0.05, N.S. $=P>0.05$. 
Table (3)

Pearson's correlation between fasting (ANGPTL4, MCP-1 and hs-CRP) and all parameters in diabetic patients (group P2).

\begin{tabular}{|c|c|c|c|c|c|c|c|c|c|}
\hline \multirow{2}{*}{ Parameter } & \multicolumn{3}{|c|}{ ANGPTL-4 (ng/ml) } & \multicolumn{3}{|c|}{$M C P-1(p g / m l)$} & \multicolumn{3}{|c|}{$\overline{C R P}(\mathrm{mg} / \mathrm{l})$} \\
\hline & $R$ & P value & Sig. & $R$ & P value & Sig. & $R$ & P value & Sig. \\
\hline Age(year) & 0.103 & 0.587 & NS & -0.222 & 0.239 & NS & 0.365 & 0.048 & $S$ \\
\hline $\begin{array}{l}\text { Duration of disease } \\
\text { (year) }\end{array}$ & 0.115 & 0.544 & NS & 0.133 & 0.484 & NS & -0.006 & 0.974 & NS \\
\hline Weight $(\mathrm{Kg})$ & 0.140 & 0.461 & NS & 0.046 & 0.810 & NS & 0.062 & 0.743 & NS \\
\hline Height $(\mathrm{cm})$ & -0.045 & 0.812 & NS & -0.153 & 0.420 & NS & -0.108 & 0.570 & NS \\
\hline BMI $\left(\mathrm{Kg} / \mathrm{m}^{2}\right)$ & 0.249 & 0.185 & NS & -0.121 & 0.525 & NS & 0.244 & 0.194 & NS \\
\hline Waist(cm) & 0.171 & 0.366 & NS & 0.140 & 0.460 & NS & 0.484 & 0.006 & HS \\
\hline $\operatorname{Hip}(\mathrm{cm})$ & 0.103 & 0.588 & NS & 0.018 & 0.606 & NS & 0.518 & 0.003 & HS \\
\hline "WHR & 0.357 & 0.053 & $\overline{\mathrm{S}}$ & -0.035 & 0.855 & NS & -0.112 & 0.556 & $\overline{\mathrm{NS}}$ \\
\hline 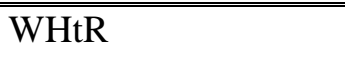 & 0.059 & 0.759 & NS & -0.046 & 0.809 & NS & 0.524 & 0.003 & $\overline{\mathrm{S}}$ \\
\hline Smoking & 0.004 & 0.984 & NS & 0.216 & 0.251 & NS & -0.008 & 0.965 & NS \\
\hline $\begin{array}{l}\text { Systolic B.P. } \\
(\mathrm{mm} \mathrm{Hg})\end{array}$ & -0.272 & 0.145 & NS & -0.091 & 0.632 & NS & 0.046 & 0.808 & NS \\
\hline Diastolic B.P. (mmHg) & -0.291 & 0.119 & NS & 0.094 & 0.62 & NS & 0.108 & 0.569 & NS \\
\hline FPG (mg/dl) & "-0.056 & 0.767 & NS & 0.18 & 0.342 & NS & -0.156 & 0.410 & NS \\
\hline Insulin( $\mu \mathrm{IU} / \mathrm{ml})$ & -0.006 & 0.975 & NS & 0.07 & 0.712 & NS & 0.374 & 0.042 & S \\
\hline HOMA2( I.R) & -0.074 & 0.697 & NS & 0.248 & 0.186 & NS & 0.237 & 0.208 & NS \\
\hline HOMA2 (S\%) & 0.063 & 0.742 & NSS & $\begin{array}{c}-0.24 \\
\end{array}$ & 0.201 & "NS & "-0.258 & 0.169 & $\overline{\mathrm{NS}}$ \\
\hline HOMA2 (B\%) & 0.107 & 0.573 & $\overline{\mathrm{NSS}}$ & $\begin{array}{c}-0.172 \\
\end{array}$ & 0.362 & $\overline{\mathrm{NS}}$ & 0.201 & 0.281 & $\overline{\mathrm{NS}}$ \\
\hline TC (mg/dl) & 0.686 & 0.000 & "HS & 0.445 & $\overline{0.014}$ & $\overline{\mathrm{S}}$ & $\overline{-0.219}$ & 0.244 & NS \\
\hline TG(mg/dl) & 0.629 & 0.000 & "HS & - -0.252 & 0.179 & NS & 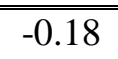 & 0.34 & NS \\
\hline HDL (mg/dl) & -0.021 & 0.913 & NS & 0.325 & 0.079 & NS & 0.198 & 0.293 & $\overline{\mathrm{NS}}$ \\
\hline$\overline{\mathrm{LDL}(\mathrm{mg} / \mathrm{dl})}$ & 0.204 & 0.281 & NS & 0.405 & 0.026 & $\bar{S}$ & -0.187 & 0.323 & NS \\
\hline VLDL (mg/dl) & 0.629 & 0.000 & $\overline{\mathrm{HSS}}$ & -0.257 & 0.171 & NS & -0.178 & 0.346 & NS \\
\hline AIP & 0.427 & 0.018 & $\bar{S}$ & -0.25 & 0.183 & $\overline{\mathrm{NS}}$ & -0.262 & 0.161 & NS \\
\hline RR1 & 0.568 & 0.001 & HS & -0.208 & 0.271 & NS & -0.263 & 0.16 & NS \\
\hline RR2 & 0.403 & 0.027 & S & 0.420 & 0.02 & $\bar{S}$ & -0.301 & 0.105 & NS \\
\hline ANGPTL-4(ng/ml) & 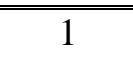 & - & - & -0.136 & 0.473 & NS & -0.454 & 0.012 & S \\
\hline MCP-1(pg/ml) & -0.136 & 0.473 & NS & 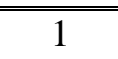 & 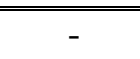 & 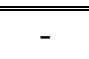 & 0.12 & 0.528 & $\overline{\mathrm{NS}}$ \\
\hline "hs-CRP(mg/l) & -0.454 & 0.012 & $\bar{S}$ & 0.12 & 0.528 & NS & 1 & - & 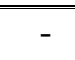 \\
\hline
\end{tabular}

H.S.: $P<0.001$, S.: $P<0.05$, N.S. $=P>0.05$. 


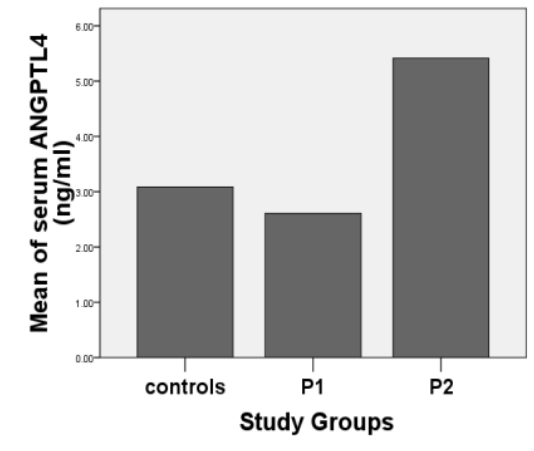

$\boldsymbol{A}$

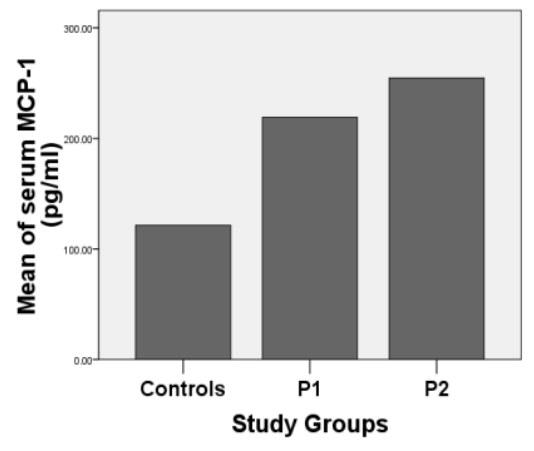

B

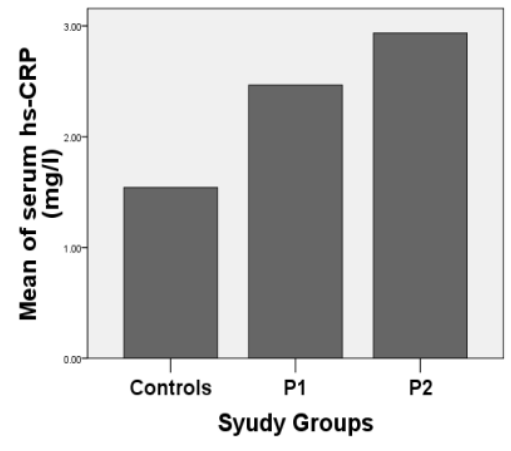

C

Fig.(1) (A-B-C) Serum ANGPTL-4, MCP-1and hs-CRP levels in diabetic patients with and without dyslipidemia (P1and P2) and control (C) groups.

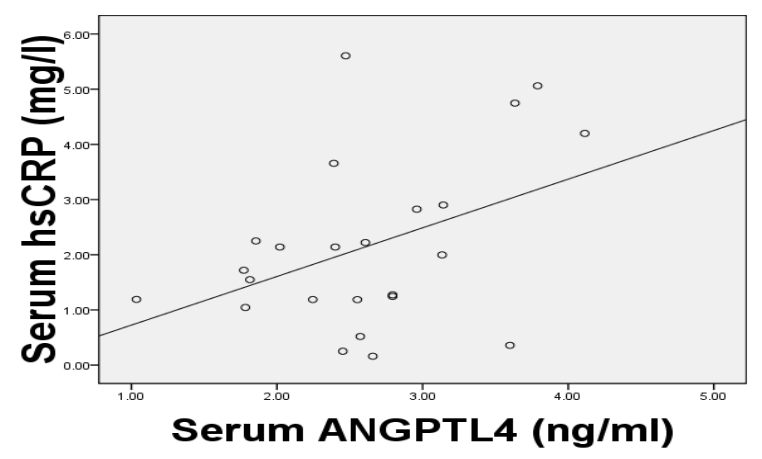

Fig.(2) Pearson correlation of fasting serum ANGPTL-4 with hs-CRP of diabetic patients without dyslipidemia (group P1).

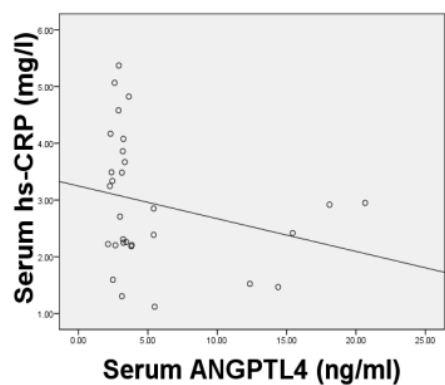

A

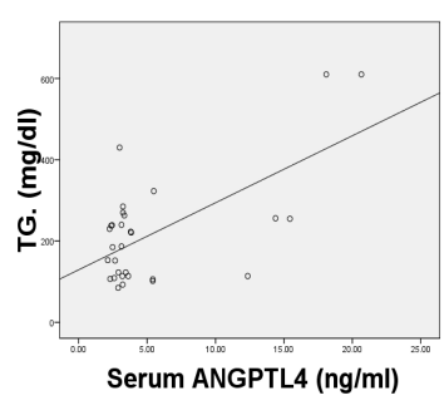

D

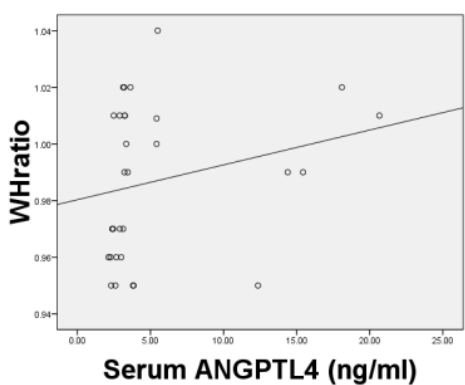

B

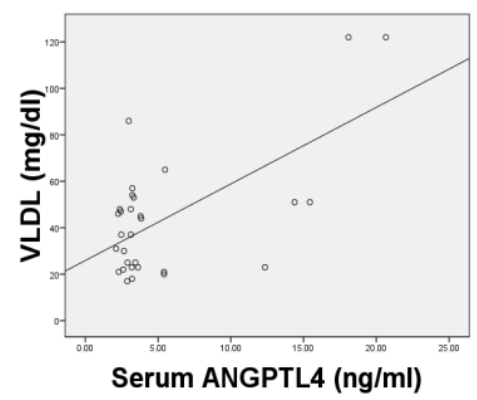

$\boldsymbol{E}$

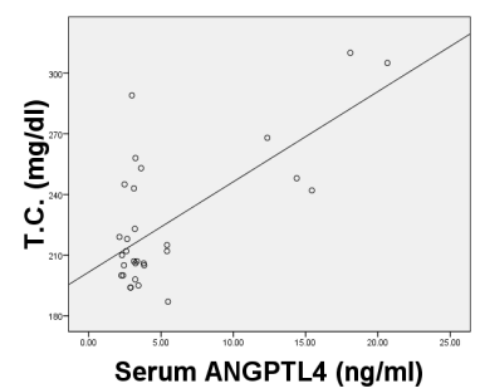

C

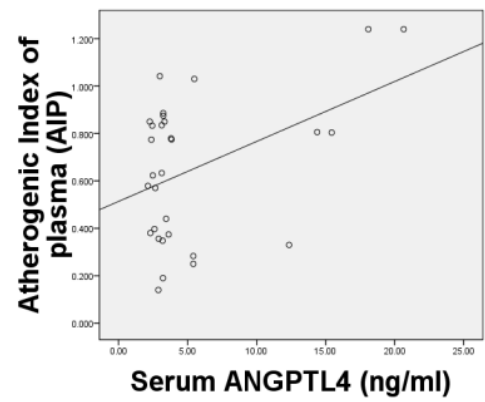

$\boldsymbol{F}$ 


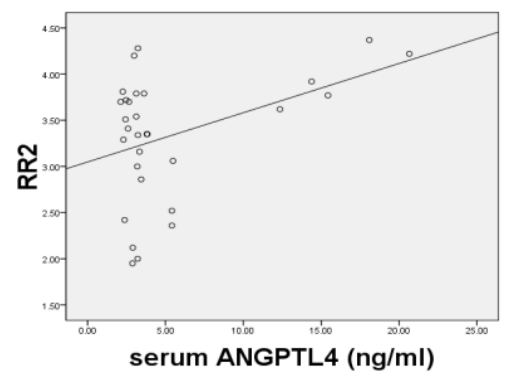

$\boldsymbol{G}$

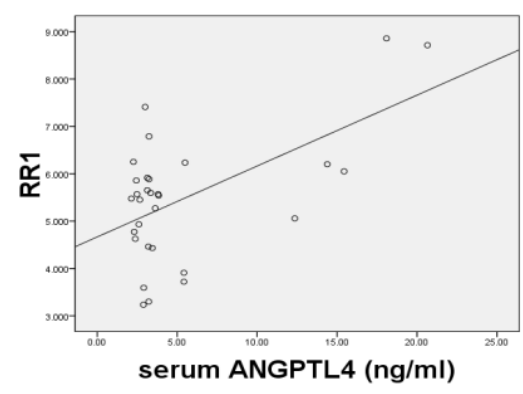

$\boldsymbol{H}$

Fig.(3) (A-B-C-D-E-F-G-H) Pearson correlation of fasting serum ANGPTL-4 with hs-CRP, TC, TG, VLDL, atherogenic parameters of diabetic patients with dyslipidemia (group P2).

\section{Discussion}

In the present study the levels of ANGPTL-4 in two groups of T2DM patients were determined; with and without dyslipidemia. It is clearly shown that the alterations in the levels of ANGPTL-4were associated with changes in the lipid profile of patients Table (1) \& Fig.(1).

Diabetic dyslipidemia is a modifiable risk factor of CVD [13]. Atherosclerosis and associated coronary heart disease (CHD) are impacted by the changes in the plasma level of lipoproteins. Elevated plasma (LDL-C) levels increase the risk of CHD, while high levels of (HDL-C) are considered to be protective [14]. In addition for high LDL there is evidence that high plasma TG is an independent risk factor for CHD [15]. A consider number of studies have been showed that the blood pressure and its progression were strong and independent predictors of incident type 2 diabetes among initially healthy persons $[16,17]$.

The results revealed a significant decrease in ANGPTL-4 of diabetes patients without dyslipidemia in comparison with group (C) Table (1) \& Fig.(1). Recently, it was shown that triglyceride-rich lipoproteins may interfere with the ability of ANGPTL-4 to inhibit LPL, a property that may also extend to LDL [18]. Also ANGPTL-4 shows no significant correlation with all parameters. Our results are in agreement with that of Yamagishi S. 2011 [19] and $\mathrm{Xu}$ et al. [20] who reported that serum ANGPTL-4 concentrations are low in patients with type 2 diabetes mellitus but not in obese individuals and there is no correlation between serum ANGPTL-4 concentrations and serum levels of triglyceride and total cholesterol suggesting that the decreased
ANGPTL-4 could be a causative factor of this diseaseand that its beneficial effect on glucose homeostasis might be useful for the treatment of diabetes. Insulin down regulates ANGPTL-4 mRNA expression, and the reduction of ANGPTL-4 mRNA by insulin is attenuated in insulin resistance [21].

ANGPTL-4 levels in T2DM patients with dyslipidemia shows positive correlation with increased levels of TC, TG, LDL, VLDL, AIP, RR1and RR2, this data is in line with Lichtenstein et al., 2010 (6), Mehta et al., 2014 [22], and with Stejskal et al., 2008 [23]. They reported a positive correlation between ANGPTL-4 levels and TG in patients with metabolic syndrome. Such elevation may be due to hepatic lipase inhibition by ANGPTL-4 which encourages the liver to increase its levels of cholesterol [24]. Robciuc et al. 2010 [25] showed that ANGPTL-4 levels were positively correlated with increase in waist-tohip ratio which is also in line with our results. We suggest that the increase in ANGPTL-4 levels in group $\mathrm{P} 2$ may be part of a protective feedback mechanism aimed at minimizing lipid overload.

Serum levels of ANGPTL-4 show positive correlation with hs-CRP levels in diabetes patients without dyslipidemia. Our results are in line with Lichtenstein. 2010 [6], which demonstrated that ANGPTL-4 protects against the severe pro-inflammatory effects of dietary saturated fat in mesenteric lymph nodes by inhibiting macrophage LPL activity, thereby reducing lipolytic release of fatty acids, macrophage foam cell formation, endoplasmic reticulum ER stress, and initiation of a marked inflammatory response. 
In contrast, serum ANGPTL-4 levels in diabetes patients with dyslipidemia showed a negative correlation with CRP. Nguanet al., 2013 [26] found that ANGPTL-4 was negatively associated with carotid artery sclerosis in subjects with the metabolic syndrome and low-grade systemic inflammation.

A hypothesis about the role of ANGPTL -4 as a new class of lipid metabolism modulator and their values could be a new key predictors of metabolic syndrome was suggested by a study of Miida et al., 2010 [27].

Accordingly, based on the present study and the results from previous studies, ANGPTL -4 pharmacologically can represent interesting candidate for the therapeutic targeting of dyslipidemia. However, further studies are still needed to clarify the influence of an altered of free fatty acid and other lipid profiles on ANGPTL-4 protein and its gene in humans as well as determine the normal value of this protein in blood plasma in order to assess ANGPTL-4 value as a new risk predictor and prognostic factor for the diagnosis of metabolic syndrome and increase atherosclerosis risk in T2DM patients.

\section{Conclusions}

There is elevated level of ANGPTL-4 in diabetic patients and positive association between ANGPTL-4 and CRP in diabetic patients without dyslipidemia could reflects the suppressive effect of ANGPTL-4 on microphage migration and chemotaxis that increase atherosclerosis risk of in T2DM patients, but this effect no longer exist in diabetic patients with dyslipidemia since two associated disease make the inflammatory state more active.

\section{References}

[1] Li S, Zhao JH, Luan J, Langenberg C, Luben RN, Khaw KT, et al "Genetic predisposition to obesity leads to increased risk of type 2 diabetes", Diabetologia, 54, $776-782,2011$.

[2] Grundy SM, Cleeman JI, Merz CN, Brewer $\mathrm{Jr}$ HB, Clark LT, Hunninghake DB, Pasternak RC, Smith Jr SC, Stone NJ, "Implications of recent clinical trials for the National Cholesterol Education Program
Adult Treatment Panel III guidelines", National Heart, Lung, and Blood Institute; American College of Cardiology Foundation, American Heart Association, 110, 227-239, 2004.

[3] Libby P, Okamoto Y, Rocha VZ, Folco E, "Inflammation in atherosclerosis: transition from theory to practice", Circ J, 74, 213220, 2010.

[4] Wang H, Eckel RH, "Lipoprotein lipase: from gene to obesity", Am J Physiol Endocrinol Metab, 297: E271-E288, 2009.

[5] Sonnenburg WK, Yu D, Lee EC, Xiong W, Gololobov G, Key B, Gay J, Wilganowski $\mathrm{N}$, Hu Y, Zhao S, et al, "GPIHBP1 stabilizes lipoprotein lipase and prevents its inhibition by angiopoietin-like 3 and angiopoietin-like 4", J Lipid Res.50, 2421-2429, 2009.

[6] Lichtenstein L, Mattijssen F, de Wit NJ, Georgiadi A, Hooiveld GJ, van der Meer R, He Y, Qi L, Køster A, TamsmaJT, Tan NS, Müller M, Kersten S, "ANGPTL-4 protects against severe proinflammatory effects of saturated fat by inhibiting fatty acid uptake into mesenteric lymph node macrophages", Cell Metab, 12, 580-592, 2010.

[7] Reynolds GD, Vance $\mathrm{RP}$, ,C- reactive protein immunohistochemical localization in normal and atherosclerotic human aortas, Arch Pathol Lab Med, 111, 265-269, 1987.

[8] Cermak J, Key NS, Bach RR, et al, "Creactive protein induces human peripheral blood monocytes to synthesize tissue factor", Blood, 82, 513-520, 1993.

[9] Pasceri V, Chang J, Willerson JT, et al, "Modulation of C-reactive protein mediated monocyte chemoattractant protein-1 induction in human endothelial cells by anti-atherosclerosis drugs", pub med, 103, 2531-2534, 2001.

[10] " Expert Committee on the Diagnosis and Classification of Diabetes Mellitus. Report of the Expert Committee on the Diagnosis and Classification of Diabetes Mellitus". Diabetes Care, 36, 567-74, 2013.

[11] Friedewald WT, Levy RI, Fredrickson DS.,"Estimation of the concentration of LDL-Cholesterol", Clin. Chem,18(6), 499515, 1972.

[12] Dobiášová M, Frohlich J., "The plasma parameter log (TG/HDL-C) as an atherogenic index: correlation with 
lipoprotein particle size and esterification rate in apoB-lipoprotein-depleted plasma (FERHDL)", ClinBiochem, 34, 583-588, 2001.

[13] Garg MK, Mohan V Kulkarni, Namita Mahalle, Sadanand S Naik, "Study of pattern of dyslipidemia and its correlation with cardiovascular risk factors in patients with proven coronary artery disease", Indian Journal of Endocrinology and Metabolism,18, 48-55, 2014.

[14] Bruckert E, Labreuche J, Deplanque D, Touboul PJ, Amarenco P., "Fibrates effect on cardiovascular risk is greater in patients with high triglyceride levels or atherogenic dyslipidemia profile", A systematic review and meta-analysis. $\mathrm{J}$ Cardiovasc Pharmacol, 57, 267-272, 2011.

[15] Kannel W.B., Vasan R.S., "Triglycerides as vascular risk factors: new epidemiologic insights", Curr. Opin. Cardiol, 24: 345350, 2009.

[16] David C, Paul M R. "Clinical significance of hs-CRP in cardio vascular disease", Biomarkers in Medicine, 2, 229-241, 2007.

[17] Cosin Aguilar J, Hernandiz. Martinez A, MasramonMorell X., "Over weight and obesity in hyper sensitive Spanish patients", The CORONARIA STUDY, Med clin (Barc), 10,129-136, 2007.

[18] Nilsson SK, Anderson F, Ericsson M, Larsson M, Makoveichuk E, Lookene A, Heeren J, Olivecrona G., "Triacylglycerolrich lipoproteins protect lipoprotein lipase from inactivation by ANGPTL3 and ANGPTL4", Biochim Biophys Acta, 1821, 1370-1378, 2012.

[19] Yamagishi S., "Cardiovascular disease in recent onset diabetes mellitus"., J Cardiol, 57, 257-262, 2011.

[20] Xu A, Lam MC, Chan KW, Wang Y, Zhang $\mathrm{J}$, et al., "Angiopoietin-like protein 4 decreases blood glucose and improves glucose tolerance but induces hyperlipidemia and hepatic steatosis in mice", Proc Natl Acad Sci U S A, 102: 6086-6091, 2005.

[21] Yamada T., Ozaki N., Kato Y., Miura Y., Oiso Y., "Insulin down regulates Angiopoietin-like protein 4 mRNA in 3T3L1 adipocytes", Biochem. Biophys, Res. Commun, 347, 1138-1144, 2006.
[22] Mehta N, Qamar A, Qu L, Qasim AN, Mehta NN, Reilly MP, Rader DJ. "Differential association of plasma angiopoietin-like proteins 3 and 4 with lipid and metabolic traits", Arterioscler Thromb Vasc Biol, 34(5): 1057-1063, 2014.

[23] Stejskal D, Karpisek M, Reutova H, Humenanska $\mathrm{V}$, Petzel $\mathrm{M}$ et al., "Angiopoietin-like protein 4: development, analytical characterization, and clinical testing of a new ELISA", Gen Physiol Biophy, 27, 59 - 63, 2008.

[24] Ridker PM, Stampfer MJ, Rifai N., "Novel risk factors for systemic atherosclerosis: a comparison of C-reactive protein, fibrinogen, homocysteine, lipoprotein (a), and standard cholesterol screening as predictors of peripheral arterial disease", JAMA, 285, 2481-2485, 2001.

[25] Robciuc MR, Tahvanainen E, Jauhiainen M, Ehnholm C., "Quantitation of serum Angiopoietin-like proteins 3 and 4 in a Finnish population sample", J. Lipid Res, 51(4), 824-831, 2010.

[26] Nguan S T, Michael M, Patrick C, Rensen $\mathrm{F}$ and Sander $\mathrm{K} \mathrm{S}$, et al, "Development Overexpression of Angiopoietin-Like Protein 4 Protects Against Atherosclerosis", Arterioscler Thromb Vasc Biol, 33,1529-1537, 2013.

[27] Miida T, Hirayama S, "Impacts of angiopoietin-like proteins on lipoprotein metabolism and cardiovascular events", Current Opinion in Lipidology, 21 (1), 7075,2010 


\section{الخلاصة}

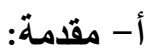

يبدأ مرض السكري مراحل تطورات تصلب الثرايين

وذلك من خلال تغيير وظيفة الخلايا البطانيه للاوعيه الدمويه, خلايا العضلات الملساء والصفيحات الدمويه, كذلك

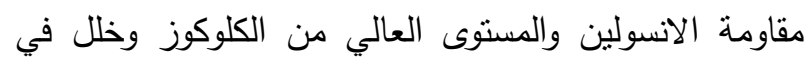

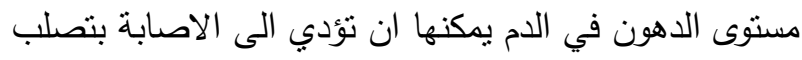

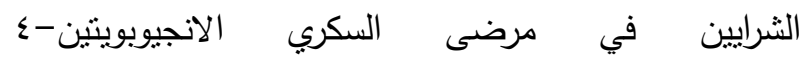
(ANGPTL-4) تنظيم مستوى الدهون, تنثيل الطاقه, تولد الاوعيه وحالات الالتهابات, تعبيره في الجسم يحفز في الكبد والقلب الآل

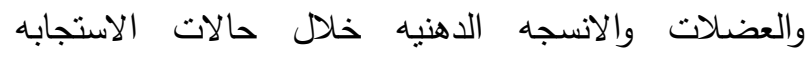

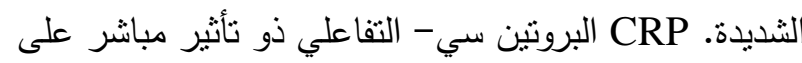
تغيير تلاصق الجزيئات في الخلايا البطانيه. وكذلك توظف مجموعة الكريات البيضاء (monocyte) الى جدار الأوعيه الدمويه من خلال تحفيز تعبير وتخليق (chemokines) خاصه ويسمى (MCP-1), كل هذه العمليات تؤدي الى

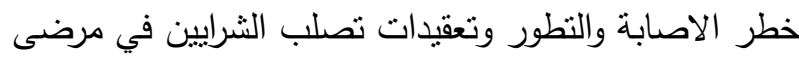

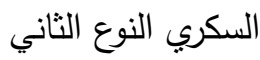

\section{ب- الهذف من الاراسة:}

هو لدراسة دور ANGPTL-4 في مرضى السكري

الذين لايهم احد اخطار الاصابه بتصلب الثرايين (خلل في دري

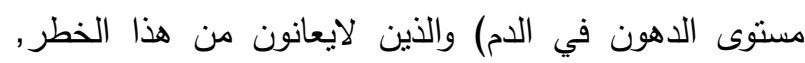
وكذللك لمعرفة العلاقه بين مستوى ع- - ANGPTL وعمليات 